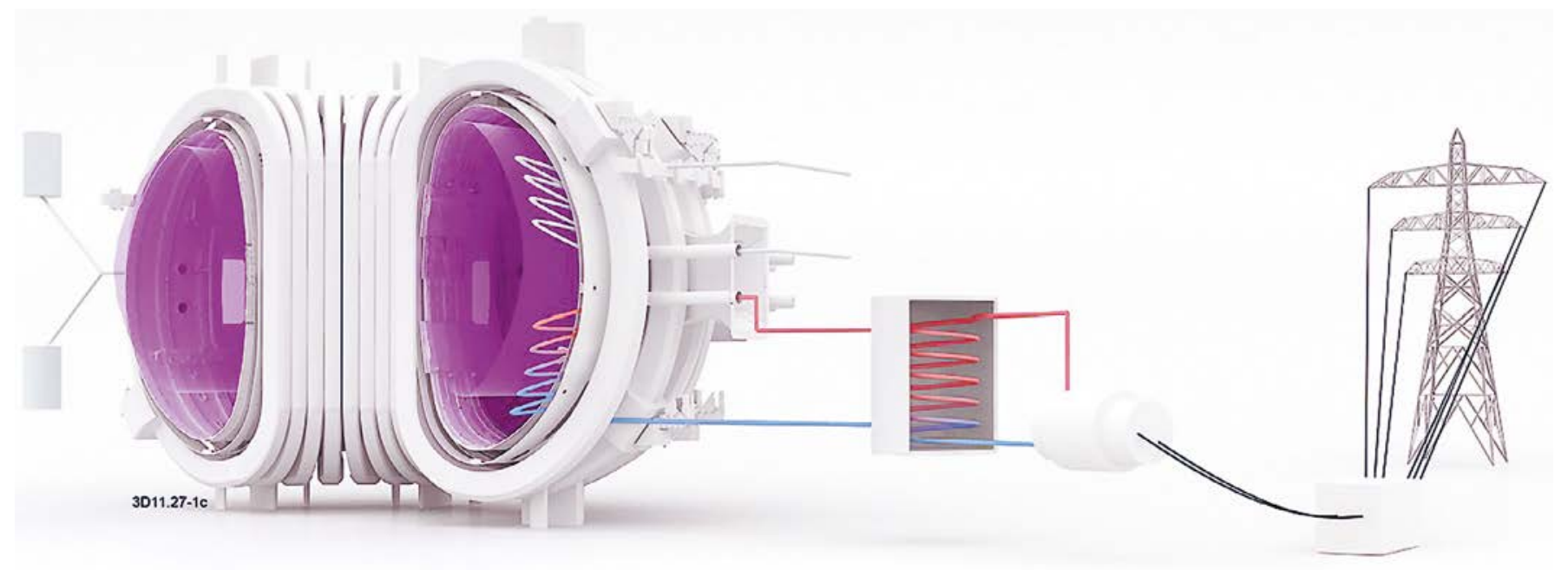

\title{
THE LIFETIME OF COMPONENTS IN A FUSION REACTOR
}

. G. Dose - DOI: https://doi.org/10.1051/epn/2021503

- University of Rome "Tor Vergata”, Department of Industrial Engineering - Via del Politecnico 1 - 00133, Rome - Italy

\section{Nuclear fusion is one of the most promising energy sources to satisfy our future needs. However, several open challenges are yet to be solved for the realisation of fusion electricity. Among the crucial issues, it is key to develop innovative solutions to increase the lifetime of the components inside a fusion reactor.}

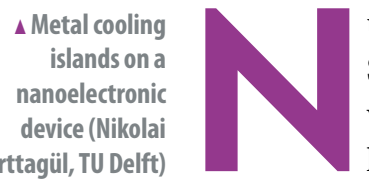
close together so that the strong nuclear force can overcome the repulsive electrostatic interaction, leading to the formation of a heavier nucleus and the release of energy. Using fusion on Earth as an energy source has been the goal of scientists from all over the world since the 1950s. Compared to other sources used for electricity production, fusion offers indeed unique advantages. Firstly, fusion is intrinsically safe since a runaway chain reaction is physically impossible inside a reactor. Moreover, the resources used as fusion fuel are available to all countries, meaning that no competition between governments would arise to ensure the supply. Most importantly, fusion benefits from a high environmental sustainability, with little to no burden left for the following generations: the fuel is virtually unlimited (available for millions of years), the amount of material resources used per TWh produced is small, no greenhouse gas is emitted during the energy production process and no long-term radioactive waste is generated. As far as waste is concerned, the reactions inside a fusion power plant would leave behind only a small amount of helium, which, being a monoatomic inert gas, is non-toxic and does not contribute to global warming. Therefore, the only waste to be managed would be due exclusively to the decommissioning of the reactor itself. The less material is needed for the overall life of the fusion plant, the less are the expenses and the environmental impact of fusion as an energy source. In this mass balance, a key role is played by the lifetime of the components operating inside a reactor, since more durable components can be substituted less frequently.

\section{The walls of a fusion reactor}

Unfortunately, coming up with a long-lasting container for a small burning star is not an easy task. The extreme environment in which a fusion reactor operates results in a combination of different loads on the walls. Stateof-the-art technology and material science are simply not mature enough to deliver a component having a lifetime equal to the overall life of the fusion plant, and consequently some parts must be replaced multiple times. The damage to the components, that determines their lifetime, depends on the different depositions that the 
burning plasma is applying on the walls, which consist of heat loads, particle loads and neutrons. The thermal energy and the particles are stopped at the wall closest to the plasma, which is covered by the so-called Plasma-Facing Components (PFCs). Neutrons, on the other hand, penetrate deeply in the machine damaging the whole volume of the reactor. Due to their location, the PFCs receive the highest damage during operation and thus these are the parts with the shortest lifetime in the reactor. In particular, they receive the thermal power that is continuously fed to the plasma to sustain the fusion reactions, the so-called plasma heating power. Due to the small heat exchange area, the heat fluxes experienced by the PFCs are extreme, starting from $\sim 1 \mathrm{MWm}^{-2}$ and reaching peaks of $20 \mathrm{MWm}^{-2}$ in special regions. To provide an idea of the order of magnitude, this power density is comparable to the one leaving the surface of the Sun, of about $60 \mathrm{MWm}^{-2}$. Such power densities result in high temperatures and steep thermal gradients that arise inside the loaded materials, leading to thermal stresses. In the PFCs, such thermomechanical loads are the main contribution to the stresses generated during the nominal operation of the reactor, and they can lead to the mechanical failure of the component by, for example, thermal fatigue or creep-fatigue. The first phenomenon is dominated by the low-cycle fatigue, which consists of the failure of the material due to the accumulation of plastic strain. An example of such behaviour can be found in Figure 1, which shows deep cracking occurring in certain PFCs, called the divertortargets, after cyclic high heat loads [1]. The creep-fatigue is a more complex and synergistic phenomenon in which creep, that is the temperature dependent irreversible deformation of a material due to the application of a monotonic stress, interacts with the thermal fatigue resulting in the acceleration of crack growth by the accumulated creep damage especially at grain boundaries or other discontinuous locations. The heat loads we have just described are applied for a sufficient time such that the thermal equilibrium is reached inside the PFCs, which is in the order of tens of seconds. However, in addition to such fluxes, also thermal shocks can occur inside a fusion reactor. These are due to instabilities (e.g., Edged localized Modes (ELMs) or Vertical Displacement Events (VDEs)) in the plasma which results in bursts of energy deposited on the wall. Since the time scales of these transients are far lower than the time scale of the thermal diffusion inside the material, only the surface layers of the PFCs will absorb all the deposited heat. Serious material degradation such as roughening or crack formation on the surface can occur, depending on the power density (which can reach $\sim 1 \mathrm{GWm}^{-2}$ for $\sim 1$ $\mathrm{ms}$ ), as well as the base temperature of the material before the transient event. Figure 2 shows an example of the surface modification that occurs when applying multiple thermal shocks to a PFC [2]. When the heat transients are

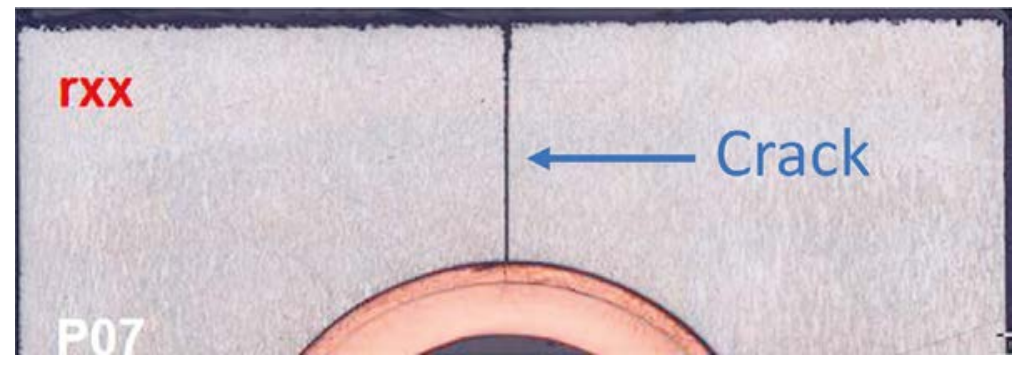

too energetic (e.g., during plasma disruptions), the energy fluences are so high and the time so short that the surface layer of the PFCs instantaneously melts and, in critical regions, evaporates. Therefore, these latter events cannot be tolerated inside a reactor and reliable mechanisms of disruption prediction and mitigation are mandatory for the design of a high-power fusion plant.

The particle fluxes experienced by the PFCs lead instead to a plethora of phenomena called Plasma-Wall Interactions (PWI). The PWI requirements put constraints on the choice of materials that can be used as an interface with the plasma, the so-called Plasma-Facing Materials (PFMs). This is the reason why the PFCs are equipped with an armour, made of a PFM, which is the part of the component interacting directly with the plasma. Among the different damages resulting from PWI, the most critical for the lifetime of components is the erosion due to sputtering. Sputtering occurs because, when charged particles collide onto a solid material, they can exchange momentum, through Coulomb collision, with the atoms inside the wall. This process can result in the ejection of atoms from the solid material, which results in an effective erosion of the wall. In PFC, the lifetime due to such process is increased by both increasing the thickness of the armor and choosing an appropriate PFM. Presently, the most promising PFM for fusion reactors is tungsten $(\mathrm{W})$, which being an element with a high atomic number is less prone to sputtering. Another detrimental effect due to PWI is the formation of a nanoscale porous structure, called "fuzz", on the surface of the PFM. Even if the effects of fuzz on the operation of a fusion reactor are yet to be quantified, this phenomenon could potentially lead to an increased possibility $\bullet \bullet \bullet$

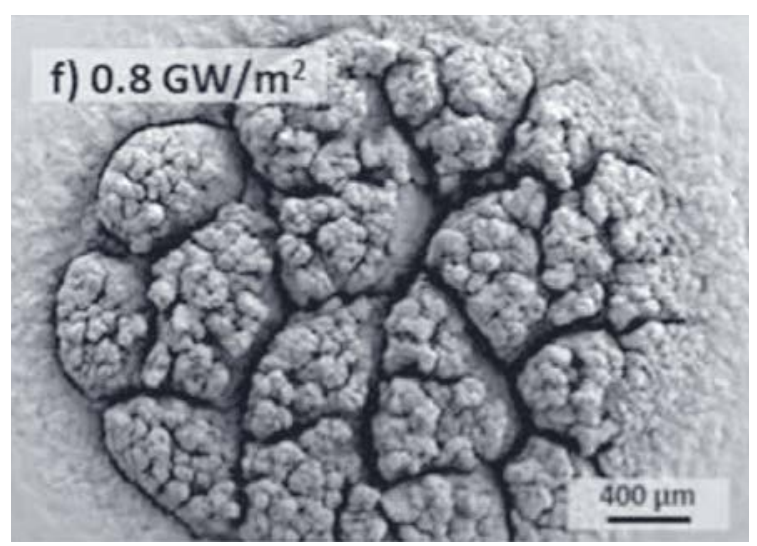

$\triangle$ FIG. 1: Deep cracking due to thermal fatigue in a divertor target [1]

4FIG. 2: Surface modification on recrystallised $W$ after $10^{5}$ heat pulses $\left(0.8 \mathrm{GWm}^{-2}\right.$, $0.5 \mathrm{~ms}$ long) [2] 


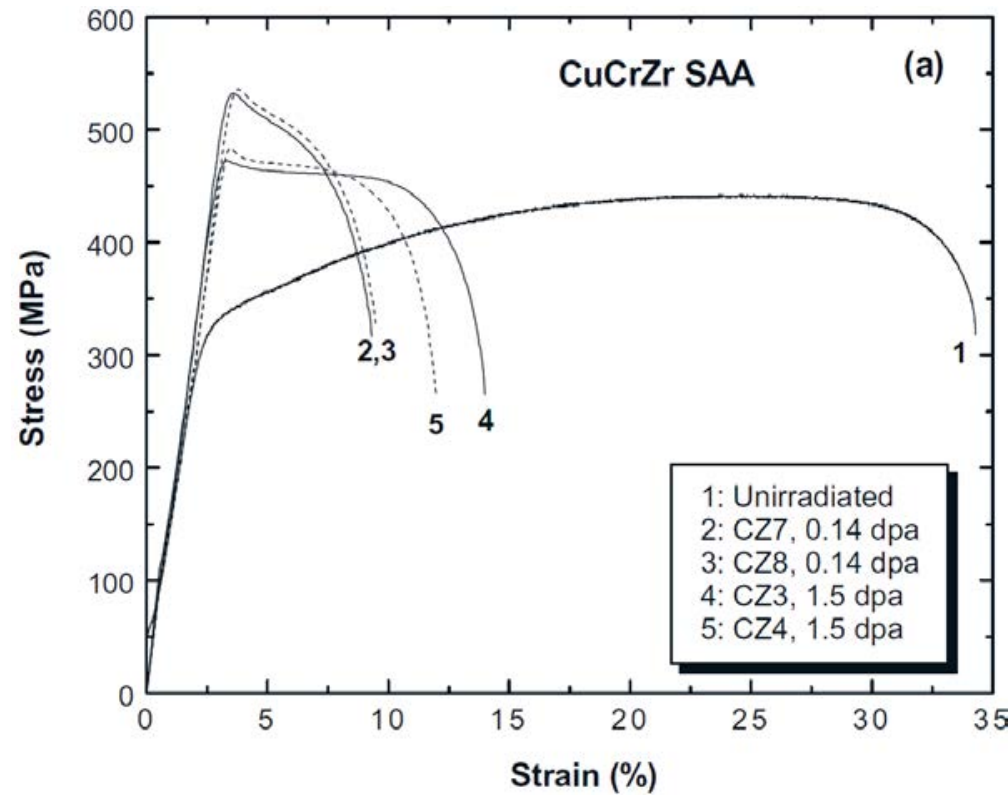

$\triangle$ FIG.3: $\bullet \bullet$ of arcing, which consists in the formation of short Engineering stress- electrical discharges occurring between the plasma and a strain curves of un-irradiated and irradiated CuCrZr [3]. Even at low displacement per atoms (dpa), the material undergoes embrittlement.

PFC. Consequently, an increased production of $\mathrm{W}$ dust and/or flakes could take place due to erosion.

\section{Neutron irradiation of the materials}

The interaction between the neutrons and the atoms inside a solid material is of nuclear nature and leads to irradiation damage. Nuclear reactions with the atomic nuclei can occur. This leads to transmutations, which consists of the generation of other elements that have different properties than the original ones, with subsequent production of light gases such as hydrogen and, most of all, helium inside the lattice material,. This leads to swelling and cluster formation worsening the mechanical properties. In addition to these processes, the lifetime of the components is also limited by the damage generated due to the so-called collision displacements. The elastic scattering, occurring between energetic neutrons impacting onto a solid component, can result in the displacement of the atoms in the walls, effectively rearranging the distribution of the nuclei in the material. Such type of irradiation damage is measured with a quantity called "displacement per atom" (or dpa) and is defined as the number of times an atom is displaced, on average, for a given neutron fluence. Due to irradiation damage, the materials change their thermophysical and mechanical properties, behaving effectively as different materials. Specifically, alloys can, at high dpa, undergo embrittlement. This is a major engineering concern, especially for the structural components which are the parts and systems that support the whole structure of the reactor. Embrittlement is a phenomenon in which a material can no longer deform plastically before ultimate failure, and suddenly fails as soon as a defect is initiated. Naturally, such a behaviour cannot be tolerated in a structural component, whose failure would result in a major damage of the whole plant. At present times, irradiation damage due to collision displacement represents the main limiting factor to the lifetime of the components of a fusion reactor able to deliver electricity to the grid. Figure 3 shows how Copper-ChromiumZirconium (CuCrZr), which is a fusion-relevant material, loses its ductility already at low dpa [3]. Such a threshold has been up to now more than sufficient for the present fusion devices. However, a reactor generating electric power from nuclear fusion would have a drastic increase in the neutron flux expected on the walls. For this reason, the development of neutron-resistant materials is one of the most active fields in fusion research. For the case of PFCs, which receive the highest neutron load, one of the potential alternatives are Reduced Activation Ferritic/Martensitic steels. However, due to their low thermal conductivity ( $\sim 10-15$ times lower than copper), they can be used only in the PFCs located at the region with heat fluxes in the range of $1 \mathrm{MWm}^{-2}$. Also, W-based composite materials are investigated, such as $\mathrm{W} /$ copper, for the region with highest thermal loads, and W/iron. Indeed, due to its high atomic number, $\mathrm{W}$ is less prone to the elastic scattering due to neutron irradiation and consequently only a small amount of dpa is generated in the material. The detrimental effects of dpa accumulation limits ultimately the lifetime of the whole fusion plant, specifically when substantial irradiation damage occurs in the parts of the reactor which cannot be substituted (e.g., vacuum vessel).

\section{The DEMO fusion plant}

For DEMO, the European fusion plant currently under design that should supply electricity to the grid in the second half of this century, the expected lifetime of the vacuum vessel is of 6 full-power years (fpy) [4]. Such a value comes from the damage threshold due to the neutron damage of the stainless steel $316 \mathrm{~L}(\mathrm{~N})$, used to manufacture the chamber. During this time, the PFCs will be remotely substituted multiple times. Specifically, the divertor units will be removed after $1.5 \mathrm{fpy}$ due to the neutron embrittlement limit, occurring at $\sim 14 \mathrm{dpa}$, of the CuCrZr used for the coolant pipes [5]. The first wall, which is the PFC covering most of the plasma-facing surface and the breeding blanket, is instead expected to operate for $\sim 5$ fpy before removal [6], since will be made of a RAFM steel, called EUROFER, which shows good mechanical behaviour even at $\sim 80 \mathrm{dpa}$ [7]. Consequently, four divertors and two breeding blankets are expected to be used during the operation of DEMO. Coming up with novel neutron resistant materials, as well as innovative engineering solutions to increase the component lifetime, would therefore impact both the economic and the environmental sustainability of nuclear fusion, since both costs and waste could be substantially reduced. The research is very active on this subject, providing new 
potential candidates [8]. Unfortunately, one still lacks the experimental validation of such novel materials since no present neutron source shares both the same energy spectrum and fluence expected in DEMO. A key facility will be IFMIF-DONES, a specific center for material testing under fusion-relevant neutron irradiation, planned to be realised before 2030 .

In conclusion, the lifetime of the components of a fusion reactor depends on the different loads experienced by the wall. Several types of damage can arise, depending on the nature of the irradiation. At present times, particular focus is dedicated to investigating synergistic effects of multiple loadings (heat and neutrons, plasma and neutrons, etc.). However, the most limiting factor to the lifetime of a fusion reactor is currently the neutron embrittlement due to the accumulation of atom displacements inside the material. In the next decades, crucial data will be provided by IFMIFDONES, which will play a key role to validate novel neutron resistant materials. .

\section{About the author}

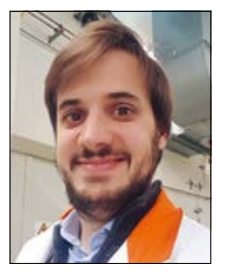

Giacomo Dose is currently a Research Fellow at the University of Rome "Tor Vergata", funded by a EUROfusion Engineering Grant awarded in 2018. He collaborates with the Italian National Agency for New Technologies, Energy and Sustainable Economic Development (ENEA) on the design and development of advanced Plasma-Facing Components for the next generation tokamaks, such as DTT and DEMO.

\section{Acknowledgements}

The author thanks Dr. Simone Noce for the useful insight into fusion neutronics, especially regarding the damage threshold of the structural materials, which are planned to be used in DEMO.

\section{References}

[1] G. Pintsuk et al., Fusion Engineering and Design 88, 1858 (2013), https://doi.org/10.1016/j.fusengdes.2013.05.091

[2] M. Gago et al., Physica Scripta T171, 014007 (2020), https://doi. org/10.1088/1402-4896/ab3bd9

[3] M. Li et al., Journal of Nuclear Materials 393, 36 (2009), https://doi.org/10.1016/j.jnucmat.2009.05.003

[4] F. Cismondi et al., Fusion Engineering and Design 124, 562 (2017), https://doi.org/10.1016/j.fusengdes.2017.03.147

[5] S. Noce et al., Fusion Engineering and Design 155, 111730 (2020), https://doi.org/10.1016/j.fusengdes.2020.111730

[6] U. Fischer et al., "Required, achievable and target TBR for the European DEMO", Fusion Engineering and Design 155 (2020) 111553, https://doi.org/10.1016/j.fusengdes.2020.111553

[7] B. van der Schaaf et al., Journal of Nuclear Materials 386-388, 236 (2009), https://doi.org/10.1016/j.jnucmat.2008.12.329

[8] G. Pintsuk et al., Fusion Engineering and Design 146, 1300 (2019), https://doi.org/10.1016/j.fusengdes.2019.02.063

\section{Electronic Instrumentation}

\section{Full floating channels with the best noise performance}

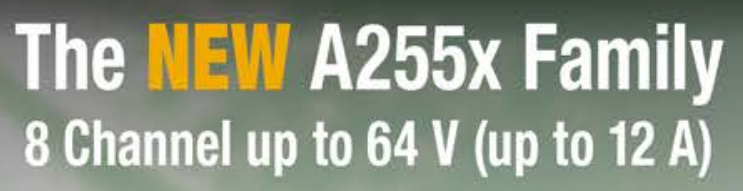

\section{For CAEN Universal Multichannel Systems, designed for front-end electronics}

\section{The power supply A255x Family is a single width} board ( 5 TE wide) that houses 8 independent Low voltage channels.

The board is available in different versions equipped with D-Sub 8-pin or with DB37 connector:
-A2551: $0 \div 8$ V output voltage and $12 \mathrm{~A}$ maximum current ( $60 \mathrm{~W}$ )

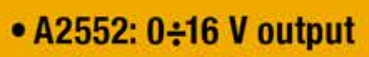
voltage and $6 \mathrm{~A}$ maximum current ( $60 \mathrm{~W})$

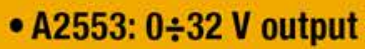
voltage and $3 A$ maximum current ( $60 \mathrm{~W})$

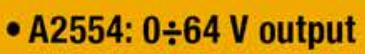
voltage and $1.5 \mathrm{~A}$ maximum current ( $60 \mathrm{~W})$

- Individual remote sense lines for voltage drop recovery

- Very Low ripple

- Channels can be parallelizable by 2 or by 4 (reaching 100 and $200 \mathrm{~W}$ )

- Under/over-voltage alert, overcurrent and max. voltage protection board and/or channel

- Software Tools for easy channel management
- Hardware interlock for

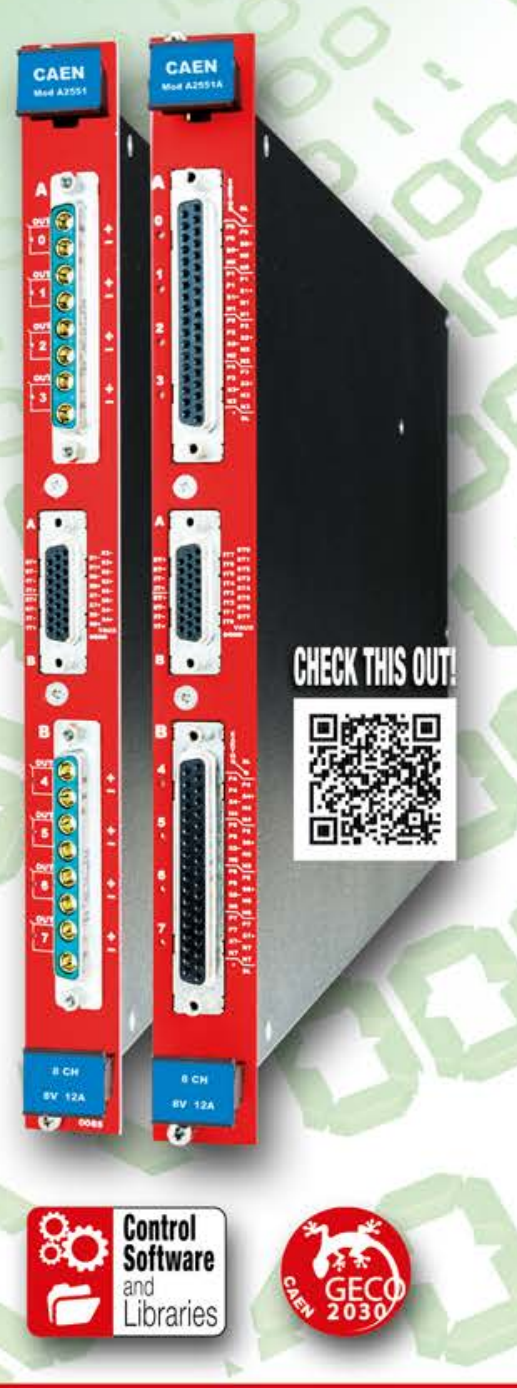

